\title{
A large sample of low surface brightness disk galaxies from SDSS
}

\author{
G. H. Zhong, ${ }^{1,2} \dagger$ Y. C. Liang, ${ }^{1} \ddagger$ L. C. Deng ${ }^{1}$ and B. Zhang ${ }^{2,1}$ \\ ${ }^{1}$ National Astronomical Observatories, Chinese Academy of Sciences, 20A Datun Road, \\ Chaoyang District, Beijing 100012, China \\ ${ }^{2}$ Department of Physicals, Hebei Normal University, Shijiazhuang 050016, China
}

\begin{abstract}
We present the properties of a large sample $(12,282)$ of nearly face-on low surface brightness disk galaxies selected from the main galaxy sample of SDSS-DR4. Those properties includes $B$-band central surface brightness $\mu_{0}(B)$, scale lengths $h$, distances $D$, integrated magnitudes, colors and some resulted relations. This sample has $\mu_{0}(B)$ from 22 to 24.5 mag $\operatorname{arcsec}^{-2}$ with a median value of $22.44 \mathrm{mag} \operatorname{arcsec}^{-2}$. They are quite bright with $M_{B}$ taking values from -18 to $-23 \mathrm{mag}$ with a median value of $-20.08 \mathrm{mag}$. The disk scale lengths $h$ are from $2 \mathrm{kpc}$ to $19 \mathrm{kpc}$. There exist clear correlations between $\log h$ and $M_{B}, \log h$ and $\log D$. Both the optical-optical and optical-NIR color-color relations show most of them have a mix of young and old stellar populations.
\end{abstract}

Keywords. Galaxies: distances and redshifts, galaxies: fundamental parameters, galaxies: photometry, galaxies: spiral, galaxies: stellar content

\section{Introduction}

Low surface brightness galaxies (LSBGs) are galaxies that emit much less light per area than normal galaxies. Yet, owing to their faintness compared with the night sky, they are hard to find. Hence their contribution to the local galaxy population has been underestimated for a long time. With the improvements of modern digital sky survey, such as the Sloan Digital Sky Survey (SDSS), the numbers of the detected LSBGs could be greatly improved. Therefore, we propose to search for a large sample of LSBGs from the SDSS database, which could help to understand the properties of LSBGs in details.

\section{The Sample}

The sample used in this work is selected from the main galaxy sample (Strauss et al. 2002) of SDSS-DR4 following the next steps:

(a) $\mathrm{fracDev}_{r}<0.25$ : this requires the galaxy having an exponential light profile since the parameter fracDev $v_{r}$ indicates the fraction of luminosity contributed by the de Vaucouleurs profile to exponential profile in the $r$-band.

(b) $b / a>0.75$ : this is selecting the nearly face-on galaxies, and $a$ and $b$ are the semimajor and semi-minor axes of the fitted exponential disk, respectively.

(c) $M_{B}<-18$ mag: keeping $M_{B}<-18$ in mind is to exclude few dwarf galaxies contained in our sample.

(d) $\mu_{0}(B) \geqslant 22.0 \mathrm{mag} \operatorname{arcsec}^{-2}$, which is following Impey et al. (2001) and is a bit lower than the first suggestion of $21.65 \mathrm{mag} \operatorname{arcsec}^{-2}$ by Freeman (1970) for LSBGs.

$\dagger$ E-mail: ghzhong@bao.ac.cn

$\ddagger$ E-mail: ycliang@bao.ac.cn 

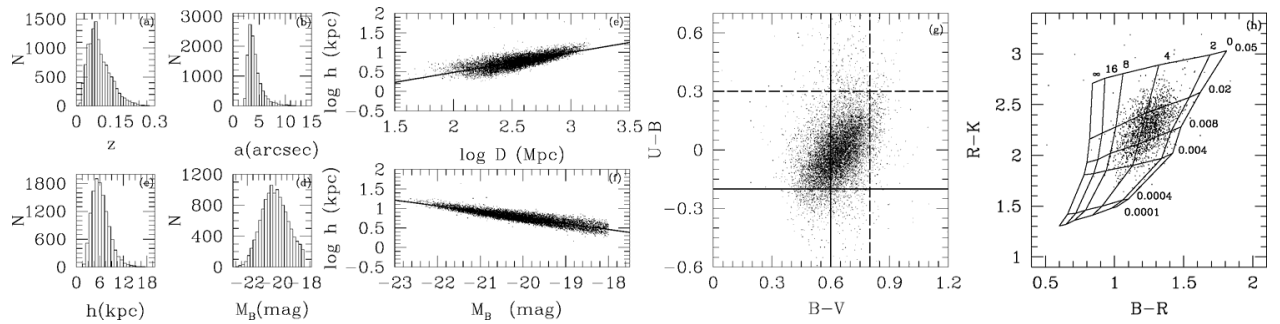

Figure 1. (a-d): histogram distributions for Sample-L: (a). the redshift, (b). the disk semimajor axis, (c). the disk radius, (d). the $M_{B} ;(\mathrm{e}-\mathrm{f})$ : correlations for Sample-L: (e). between $\log h$ and $\log D$, (f). between $\log h$ and $M_{B} ;$ (g). optical colur-color diagram of Sample-L; (h). optical-NIR color-color diagram of Sample-L2.

(e) Cross-correlation with 2MASS NIR photometry: after doing cross-correlation with 2MASS photometry, 1,878 galaxies are selected.

With step $(\mathrm{a}-\mathrm{d})$, we have selected a large sample of nearly face-on low surface brightness disk galaxies (12,282 galaxies, named Sample-L). Figure 1(a-d) show the histogram distributions of some property parameters of Sample-L. The sample selected by crosscorrelation with 2MASS was named as Sample-L2.

\section{Results and conclusions}

In Fig. 1e, we show the correlation between $\log h$ and $\log D$ for Sample-L, which can be fitted by a least-square fit as: $\log h=0.511( \pm 0.004) \log D-0.536( \pm 0.010)$, with standard deviation of 0.10 dex. This relation obviously show the selection effect of observations. Fig. If shows the correlation between $M_{B}$ and $\log h$ for Sample-L. It can be fitted by a least-square fit as: $\log h=-0.150( \pm 0.0007) M_{B}-2.245( \pm 0.014)$, with small standard deviation of 0.070 dex. This correlation suggests there are limits on both low luminosity and large scale length galaxies, otherwise such LSBGs are very rare (Impey et al. 2001).

Figure $1 \mathrm{~g}$ shows $U-B$ vs. $B-V$ diagram of Sample-L, the solid and dashed lines are suggested by O'Neil et al. (1997a, b) to define the "very blue" and "very red" LSBGs. Figure $1 \mathrm{f}$ shows the trends in $B-R$ and $R-K$ optical-NIR diagram for Sample-L2. The overplots are the stellar population synthesis grids obtained by Bell et al. (2000) using the models of Bruzual \& Charlot (2003), where the horizontal lines refer to the different metallicities and the vertical lines refer to the different characterized e-folding time scale of their star formation. Both the optical-optical and optical-NIR color-color relations show that most of the sample LSBGs have a mix of young and old stellar populations.

\section{Acknowledgements}

We thank the NSFC grant support under Nos. 10403006, 10673002,10573022 and the National Basic Research Program of China (973 Program) No.2007CB815404, 06.

\section{References}

Bell, E. F. \& de Jong, R. S. 2000, MNRAS 312, 497

Bruzual, G. \& Charlot, S. 2003, MNRAS 344, 1000

Freeman, K. C. 1970, ApJ 160, 811

Impey, C., Burkholdert, V., \& Sprayberry, D. 2001, AJ 122, 2341

ONeil, K., Bothun, G. D., \& Cornell, M. 1997a, AJ 113, 1212

ONeil, K., Bothun, G. D., Schombert, J., Cornell M. E., \& Impey, C. D. 1997b, AJ 114, 2448

Strauss, M., et al. 2002, AJ 124, 1810 